\title{
Codiseño de Objetos de Aprendizaje OA como estrategia de capacitación a docentes de Educación Superior
}

\section{Codesigning Learning Objects as a strategy of training for Higher Education teachers}

Evelyn Diez-Martínez; evelyn@uaq.mx

Rosa Alejandra Morales-Velasco; rosa.alejandra.morales@uaq.edu.mx

Universidad Autónoma de Querétaro (México)

\section{Resumen}

Los Objetos de Aprendizaje (OA) son una opción para crear contenido digital educativo. Diseñarlos implica procesos complejos requiriendo de los autores competencias que exceden las exclusivamente disciplinares. Para identificar las opiniones iniciales y finales de 12 docentes de universidad acerca de la dificultad o facilidad que el diseñar un OA les implicaba, se les impartió un curso sobre cómo diseñar un OA, con una metodología específica (DICREVOA 0.2) y se les proporcionó acompañamiento como codiseño de estos OA, lo anterior como estrategia para identificar posibles cambios en sus opiniones después del curso. Los resultados muestran que tareas relacionadas con aspectos pedagógicos ligadas a su praxis les son más fáciles que aquellas requiriendo de aspectos tecnológicos, ya que menos del 50\% concretaron el guion del diseño de la interfaz. Sin embargo, ambos aspectos se beneficiaron del curso apareciendo diferencias entre las opiniones iniciales y finales de los docentes autores sobre sus competencias al diseñar un $\mathrm{OA}$. Esto manifiesta la necesidad de ofrecer en las instituciones de educación, programas de formación en estrategias de codiseño y diseño de recursos educativos digitales, para responder a las transformaciones actuales en la transmisión del conocimiento y apunta a la importancia de seguir realizando investigación al respecto.

\section{Palabras clave:}

Objeto de Aprendizaje, codiseño educativo, educación superior, competencias digitales.

\begin{abstract}
Learning Objects (LOs) are an option to create digital educational content. Designing them involves complex processes that require from authors competences that exceed disciplinary ones. In order to identify the initial and final opinions of 12 university teachers about the difficulty or ease that designing an LO implied, they received a course on how to design an LO, using a specific methodology (DICREVOA 0.2) and were given accompaniment in form of codesign of these $L O$, the previous as a strategy to identify possible changes in their opinions after the course using the mentioned methodology above. The results show that the tasks related to pedagogical aspects linked to their praxis are easier for them than those requiring technological aspects, since less than 50\% completed the script of the interface design. However, both aspects were benefited from the course, because of differences appearing between the initial and final opinions of teachers as authors on their competences of designing an $L O$. This shows the need for educational institutions to offer teachers training courses on strategies to codesign and design digital educational resources, to respond to the current transformations in the transmission of knowledge and highlights the importance of further research on the subject.
\end{abstract}

\section{Keywords:}

Learning Object, educational co-design, higher education, digital competences. 


\section{INTRODUCCIÓN}

En el contexto actual de una pandemia mundial generalizada por el COVID19, no hay opciones para los procesos educativos más que la virtualidad, incrementándose la relevancia de que los docentes cuenten con competencias digitales (Martínez-Garcés y Garcés Fuenmayor, 2020). Los cursos masivos abiertos en línea (MOOC) reúnen todas las condiciones necesarias para ofrecer una posible solución a la ineludible y apremiante necesidad de formación inicial y continua del profesorado en el área de creación de contenidos, como parte de la competencia digital (Gordillo, López-Pernas y Barra, 2019).

Dentro del diseño educativo de este tipo de recursos los Objetos de Aprendizaje (OA), se erigen como una de las tendencias en el campo de la educación mediada por las TIC. Estudios recientes como el de Barba, De la Calle, Calderón y Ramos, (2019), han establecido cómo la capacitación a docentes mediante cursos en el diseño de OA, contribuye a elevar su nivel de conocimiento y actualización en recursos didácticos con sustento en las TIC, al comparar un grupo experimental que diseñó un OA con un grupo control que no lo diseñó. En el presente estudio se diseñó un curso para docentes universitarios, como estrategia de análisis de la metodología DICREVOA 2.0 y del acompañamiento y codiseño, para propiciar el aprendizaje de competencias y actualización de docentes en el diseño de Recursos Educativos Digitales (RED) tales como los OA.

Aunque existe una gama de definiciones de OA, la presente investigación adopta la definición de Maldonado, Bermeo y Vélez (2017), quienes mencionan que un OA es:

"Una unidad digital independiente, cuya estructura está formada por un objetivo de aprendizaje específico, un contenido, un conjunto de actividades y una autoevaluación. Puede ser reutilizado en diferentes contextos tecnológicos (repositorios, entornos virtuales de enseñanza y aprendizaje) y educativos, además cuenta con metadatos que propician su localización." (p.10)

El proceso de diseño de un OA es complejo ya que implica procesos que exigen de los autores diversas competencias que exceden las exclusivamente disciplinares, ya que requiere de la conjunción de varios conocimientos y habilidades acerca del diseño instruccional, el diseño de contenidos multimediales así como sobre diseño de interfaces, interoperabilidad tecnológica, etc. De manera general existen dos tendencias o modelos para el diseño de un OA, el primero se visualiza como un modelo de trabajo interdisciplinario de un equipo de especialistas. El segundo es un modelo en donde el autor está a cargo de la mayoría del proceso de construcción del OA.

Que la construcción de un OA se geste bajo un modelo u otro, depende de las circunstancias del contexto, por ejemplo: de la existencia de recursos humanos necesarios (especialistas), de docentes con las competencias digitales requeridas, del tipo de OA a desarrollar, de la apropiación que tenga la comunidad académica del concepto de OA, etc. Sin embargo, en cualquiera de los modelos, la figura del docente siempre debe estar presente, pues es el experto en la disciplina del tema a tratar. 
El desafío se presenta cuando el docente está a cargo del proceso, que en la mayoría de los casos no cuenta en su entorno o institución con un equipo multidisciplinario que le de el apoyo necesario para crear un recurso educativo de calidad. Es aquí cuando además de ser especialista en su área disciplinar, tiene que serlo en otras y enfrentarse a un proceso complejo haciéndolo generalmente de manera empírica y poniendo en marcha estrategias y herramientas a su consideración; esfuerzos que no siempre están dirigidos a construir un OA óptimo (Menéndez, Prieto y Zapata, 2010). Ante esta realidad, sin duda la capacidad que tenga el docente como autor de un $\mathrm{OA}$, es fundamental para obtener un recurso de calidad, situación que impacta directamente en el aprendizaje mediado por este objeto tecnológico.

\subsection{Competencias digitales docentes en el diseño de recursos educativos digitales}

La presencia inminente de las TIC ha obligado a cambiar la intermediación entre los contenidos pedagógicos y el estudiante (Trujillo, 2013), hecho ligado no solo a la tecnología como instrumento, sino también a las diversas modificaciones actuales en la educación. Esto se relaciona con las nuevas prácticas de enseñanza y de aprendizaje, lo que transforma el papel tradicional del docente como único emisor y lo convierte en docente digital con la consigna de guiar el aprendizaje del estudiante a través de diversas estrategias.

Hoy en día es difícil imaginar a un docente como un expositor de temas frente a los estudiantes y así también a un estudiante que es receptor, cumpliendo solo con anotar y memorizar dicho conocimiento. Por esto, el docente al estar informado y capacitado puede apropiarse de las nuevas herramientas y nuevos lenguajes para incorporarlos al proceso educativo, pero lo más importante, puede ser capaz de valorar el impacto de las nuevas tecnologías en sus estudiantes (Ogalde y González, 2008).

Sin duda, este cambio de paradigma obliga a pensar en la capacidad del docente, pues de antemano se cuenta con estudiantes que nacieron con la tecnología, y viven un proceso de formación fuera del aula en donde interactúan con ella de manera natural. Por lo tanto, como lo señala Cabero (2014), es el docente quien sufre un proceso de adaptación, pues debe transformar su clase con cualquier elemento que le es ofrecido. Ante esto, la tarea ineludible del docente es profundizar su conocimiento en el mundo de las TIC, apropiarse de sus lenguajes y reflexionar sobre "la forma en que cada una de ellas puede ser utilizada para transmitir conocimiento y habilidades, de acuerdo con las principales teorías pedagógicas" (Ogalde y González, 2008, p.7).

La formación de un docente en este siglo implica que esté involucrado con varias competencias que reflejen su experiencia tanto en el área de conocimiento en la que es especialista, como en aplicaciones tecnológicas que fomenten aprendizajes autónomos (Casadei y Barrios, 2016).

De manera específica, la tarea de diseñar recursos y materiales digitales para apoyar las tareas de enseñanza y de aprendizaje con intenciones pedagógicas (RED), hasta hace algunos años solo se llevaba a cabo por personas que tenían acceso a infraestructura y recursos sofisticados. Actualmente es relativamente más sencillo incursionar en el diseño de RED que presenten versatilidad, pues la Internet ha puesto a disposición un sinfin de aplicaciones para 
producirlos de manera atractiva, con la posibilidad de cruzar contenido, actividades de aprendizaje y procesos comunicacionales (Ogalde y González, 2008; Casadei y Barrios, 2016).

Diseñar OA por parte del docentes es una tarea ardua, pues para lograr un objetivo de aprendizaje a través de un contenido digital, se requiere el diseño de actividades y autoevaluaciones que pongan en evidencia lo que el estudiante logró, así como que se exploren todos los lenguajes multimedia. Al diseñar un OA el docente adquiere una serie de competencias relacionadas con la producción de materiales digitales enfocados a procesos de enseñanza y de aprendizaje, diseño instruccional, búsqueda, selección y evaluación de RED, diseño multimedial para crear la interfaz y el diseño de navegación, publicación en la Web, manejo de derechos de autor y licenciamiento entre otras. Todas estas le posibilitan una autonomía en la creación y utilización de transmisión virtual del conocimiento que resulta tan importante en la actualidad.

\subsection{Diseño de Objetos de Aprendzaje centrado en el docente como autor.}

Entre las metodologías existentes para diseñar OA están aquellas que privilegian al Diseño Instruccional, y otras al desarrollo del software, así también están las que requieren de un equipo multidisciplinario para el diseño del OA y algunas que conceptualizan al docente como autor.

Cuando el docente necesita diseñar un OA, se encuentra en un escenario desafiante. Primeramente, por la tarea de identificar y adoptar una metodología adecuada a sus intereses $y$, en segundo lugar, por las competencias pedagógicas y tecnológicas que le demanda (Maldonado, Sanz, Fernández-Pampillón y Astudillo, 2015).

En un trabajo realizado por Morales-Velasco y Diez-Martinez (2020) que hizo una revisión de diez metodologías para el diseño de OA publicadas en Latinoamérica, se reportó que la metodología DICREVOA 2.0 (Maldonado et al., 2017) fue la que más aspectos pedagógicos y tecnológicos integró conforme a los parámetros de Morales (2005), así como elementos básicos y características propias de un OA marcadas por CODAES (2015), y que principalmente es una de las pocas metodologías que integra al docente como autor de OA.

Tomando lo anterior en consideración, y el que nuestros objetivos consistían en identificar el impacto de un curso sobre diseño de un OA, como estrategia de análisis de metodologías específicas y del acompañamiento y codiseño de estos materiales digitales en docentes de educación superior, se trabajó específicamente con la metodología DICREVOA 2.0. La misma está compuesta por 5 fases con una secuencia de 16 tareas consecutivas para guiar al docente en el desarrollo de un OA. A continuación, solo se enumeran aquellas tareas y competencias didácticas y digitales incluidas en la metodología mencionada, abordadas en el curso y reportadas en los datos y resultados del presente trabajo. Para mayor precisión debe consultarse la descripción completa de DICREVOA 2.0 en Maldonado et al. (2017).

\section{Fase 1 - ANÁLISIS}

Tarea 2: Definir un único objetivo de aprendizaje del OA.

\section{Fase 2 - DISEÑO INSTRUCCIONAL}

Tarea 3: Definir qué contenidos se van a incluir en el OA y cuáles no. 
Tarea 6: Definir tipo de actividades del OA según el estilo de aprendizaje de los estudiantes (iniciales y breves-estilo activo-, para tomar conciencia del tema-estilo reflexivo-, para generar nuevas hipótesis-estilo teórico- o actividades que promuevan la transferencia de conocimientos-estilo pragmático-).

\section{Diseño Multimedial}

Tarea 8: Crear el diseño de la interfaz (pantalla) del Objeto de Aprendizaje según el tema a tratar.

Tarea 9: Esquematizar el flujo de la información o también llamada navegación del OA (de manera lineal simple, lineal ramificada, jerárquicamente o en red).

\section{Fase 3 - IMPLEMENTACION}

Tarea 11: Ensamblar tecnológicamente el Objeto de Aprendizaje integrando el diseño de la interfaz y el diseño de la navegación antes definidos, para hacer un OA funcional y navegable.

\section{Fase 5- PUBLICACIÓN}

Tarea 15: Empaquetar (comprimir) el OA en un estándar reconocido en todos los Entornos Virtuales de Enseñanza Aprendizaje sobre los que se desee publicar el OA.

\section{MÉTODOS}

Se conformó una muestra intencional mediante una convocatoria en una universidad pública en Querétaro, México, a través de la cual se logró captar la participación de 12 docentes de Educación Superior de disciplinas diversas, que siguieron y terminaron el curso. De los participantes 3 tenían entre 1 y 5 años de experiencia docente, 6 entre 5 y 10 años y 3 entre 15 y 20 años. Como requisito de aceptación al curso, se dispuso que los docentes hubieran diseñado o utilizado en su praxis cualquier tipo de recurso educativo digital. Para acompañar y guiar a los docentes en el proceso de diseñar un OA, se construyó un curso de formación docente a distancia con una duración de 25 horas distribuidas en 2 semanas de trabajo, compuesto por cinco módulos, que incluyeron en su conjunto las 16 tareas específicas propuestas por la metodología DICREVOA 2.0 (Maldonado et al., 2017). De estas tareas en el presente trabajo solo se reportan los resultados de algunas. En dicha metodología para cada una de las fases se presenta información teórica y práctica pertinente para apoyar a los autores-docentes y plantillas específicas para desarrollar cada tarea definidas por Maldonado et al. (2017). Aunque la metodología DICREVOA 2.0 es clara y permite que el docente siga el diseño de manera autónoma, durante el curso y con el fin de proporcionar acompañamiento como codiseño a los participantes, se les proporcionaron enlaces a repositorios de OA generados en otras instituciones de educación para permitirles apreciar estos recursos ya terminados y publicados. Asímismo, al finalizar cada tarea y el producto correspondiente, el instructor del curso enviaba comentarios positivos o indicaciones de cómo mejorar el producto implicado en la tarea.

\section{Cuestionario de opinión.}

Para identificar la opinión de los participantes en cuanto a su nivel de dominio o dificultad en cada una de las tareas para diseñar un OA, se diseñó un cuestionario que sirvió como pre test. Dicho cuestionario estuvo conformado por 16 enunciados acerca de las tareas necesarias para diseñar un OA, según la metodología DICREVOA 2.0. Para ello se presentó una escala con las opciones de respuesta: no lo sé, muy difícil, difícil, fácil y muy fácil. Por posibilidades de 
extensión y relevancia los resultados aquí reportados corresponden únicamente a la valoración de 7 de los enunciados correspondientes a las tareas de: -Definir un único objetivo de aprendizaje del OA, -Definir cuáles contenidos se van a incluir en el OA y cuáles no, -Definir tipo de actividades del OA según el estilo de aprendizaje de los estudiantes, -Crear el diseño de la interfaz (pantalla) del Objeto de Aprendizaje según el tema a tratar, -Esquematizar el flujo de la información o también llamada navegación del OA (de manera lineal simple, lineal ramificada, jerárquicamente o en red), -Ensamblar tecnológicamente el OA integrando diseño de la interfaz y diseño de la navegación antes definidos, para hacer un OA funcional y navegable, y-Empaquetar (comprimir) el OA en un estándar que sea reconocido en todos los Entornos Virtuales de Enseñanza Aprendizaje sobre los que se desee publicar el OA.

Como complemento, se agregaron dos preguntas abiertas con el fin de ampliar y contrastar los resultados obtenidos mediante las preguntas cerradas del cuestionario. Con el fin de establecer si el curso modificaba las opiniones de los docentes participantes, el mismo cuestionario se aplicó como pos test al finalizar el curso.

\section{RESULTADOS}

Los resultados fueron obtenidos estableciendo porcentajes de frecuencia de respuesta ante las diferentes opciones presentadas en cada una de las preguntas para el pre test y el post test.

Los argumentos de los participantes ante las preguntas abiertas inicial y final son presentados en una tabla que permite la comparación entre los mismos al iniciar el curso y al finalizar el mismo. Por cuestiones de posibilidades de extensión del texto, se presentan solo los resultados de algunas tareas en cada una de las fases de la metodología utilizada, las cuales resultaron significativas al contrastar las opiniones y percepciones de los docentes al inicio y al final del curso.

\section{FASE DE ANÁLISIS}

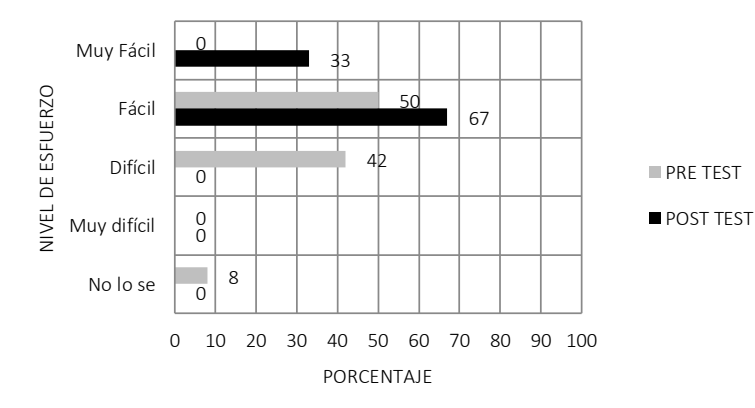

Figura 1.Definir un único objetivo de aprendizaje.

La figura 1 permite apreciar que en el pretest el $42 \%$ de los participantes señalaron que elaborar un objetivo de aprendizaje les resulta difícil, respuesta interesante cuando se considera que dicha tarea es esencial en la elaboración de los contenidos de cualquier programa de estudios y los años de experiencia en docencia de los participantes. La figura muestra como este tipo de respuesta ya no aparece en el postest y el 100\% de respuestas se agrupan en que elaborar un objetivo de aprendizaje ya les es o fácil o muy fácil a los participantes.

FASE DE DISEÑO INSTRUCCIONAL 


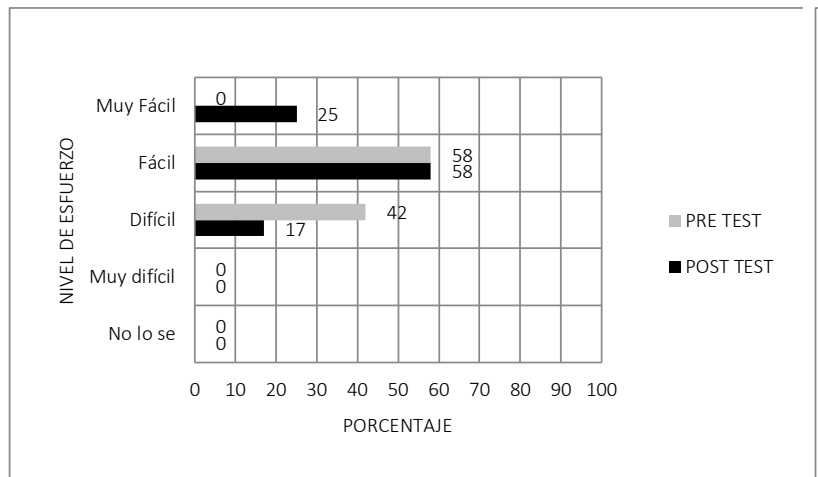

Figura 2.Definir contenidos

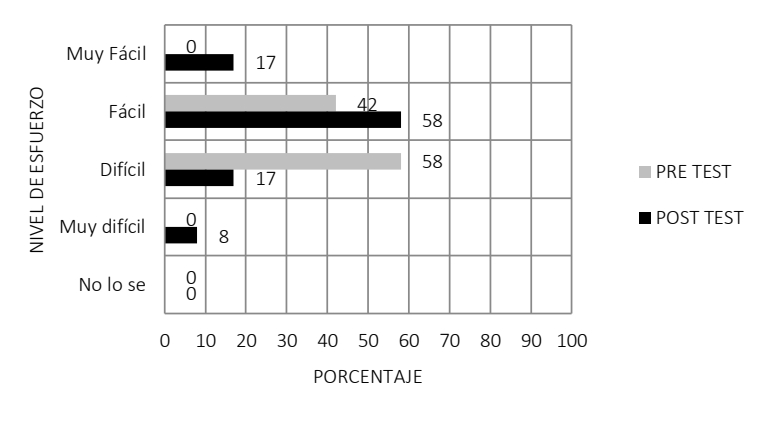

Figura 3. Definir tipo de actividades.

Las figuras 2 y 3 se relacionan con las tareas concernientes al diseño instruccional de los recursos educativos. La figura 2 permite apreciar que las respuestas del pretest muestran que los participantes tienen dificultad con definir los contenidos de aprendizaje que incluyen en los recursos educativos que diseñan. Aunque las respuestas mejoran en el postest, todavía un número de participantes considera difícil el definir los contenidos de sus recursos.

La figura 4 muestra que los participantes en un 58\% señalan que la definición del tipo de actividades en el diseño de sus recursos educativos es una tarea difícil y aún habiendo una mejoría en las respuestas del postest en cuanto a que esta tarea se volvió fácil y muy fácil, todavía existen respuestas que hablan de la dificultad de efectuar la tarea. Cabe señalar que las tareas requeridas son la esencia del diseño que un docente debe realizar para cada uno de sus cursos independientemente que estas impliquen o no aspectos tecnológicos, y los datos indican que los docentes opinan que tienen dificultades para llevarlas a cabo. Por otra parte los datos también señalan que los contenidos del curso beneficiaron a los profesores en cuanto a sus opiniones sobre las competencias incluidas en el diseño instruccional que ellos se reconocen a este respecto.

Probablemente los docentes sí incluyen objetivos de aprendizaje en forma de enunciados en sus cursos, definen sus contenidos y el tipo de actividades correspondientes, aunque muchos de ellos nunca han recibido información de planeación curricular ni conocen el diseño instruccional correspondiente. El hecho de cambiar totalmente sus opiniones al respecto después de recibir información pertinenente, muestra la imperiosa necesidad de capacitación institucional a este respecto.

Resultados semejantes han sido reportados en un estudio realizado por Camargo-Escobar y Pardo-Adames (2007) cuyo objetivo fue el diseño, construcción y validación de un instrumento para evaluar las competencias docentes de los profesores de pregrado de una universidad en Colombia. El instrumento evaluaba diversas competencias básicas para el ejercicio de la función docente: planificación curricular, utilización adecuada de diseño metodológico y organización de actividades de enseñanza, competencia científica o disciplinar y algunas otras no representadas en los datos del presente trabajo. Utilizando una muestra de 20 docentes en activo, los autores encontraron que un alto porcentaje de los profesores presentaron un nivel de competencias por debajo del esperado en la mayoría de los ítems del instrumento.

FASE DE DISEÑO MULTIMEDIAL 


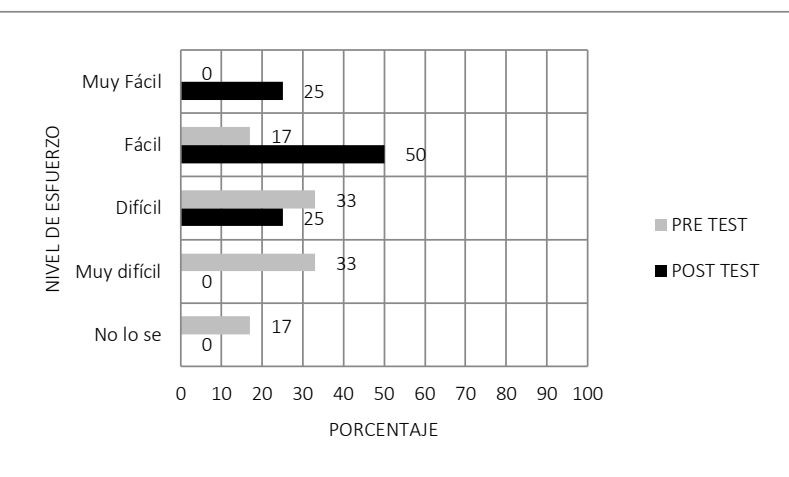

Figura 4.Crear el diseño de la interfaz.

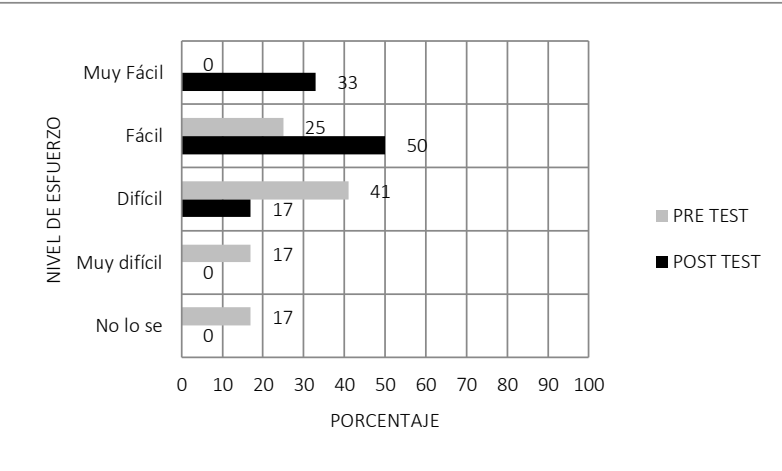

Figura 5.Esquematizar flujo de la información.

- La figura 4 incluye las opciones seleccionadas por los participantes acerca de la creación de la interfaz o presentación en pantalla del tema a tratar en el OA. Se aprecia claramente que en el pretest muy pocos son los docentes que opinan que realizar esto es fácil, sino más bien que les es muy dificil, difícil, o no saben a qué se hace referencia.

- La figura 5 muestra las opiniones de los participantes ante la tarea de esquematizar el flujo de la información o también llamada navegación del OA (de manera lineal simple, lineal ramificada, jerárquicamente o en red). Como puede apreciarse muy pocos son los participantes que reconocen esto como una tarea fácil. En ambas figuras 4 y 5 en el postest la situación cambia considerablemente mostrando los efectos del curso y el acompañamiento en el codiseño proporcionado por el instructor.

FASE DE IMPLEMENTACIÓN

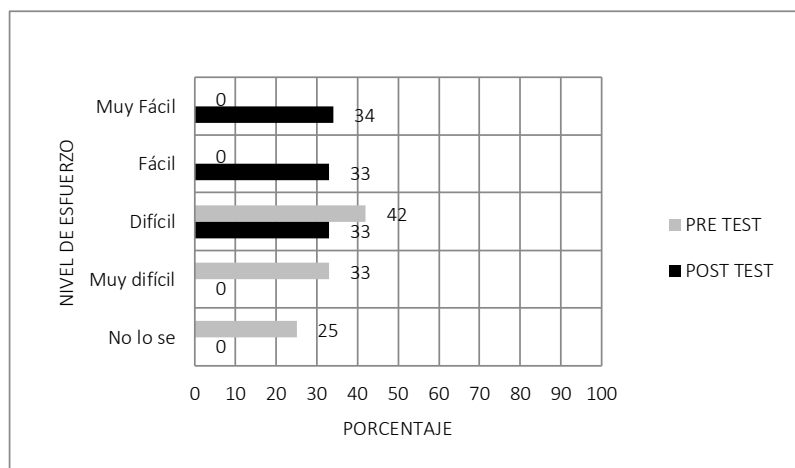

Figura 6. Ensamblar tecnológicamente el OA.

\section{FASE DE PUBLICACIÓN}

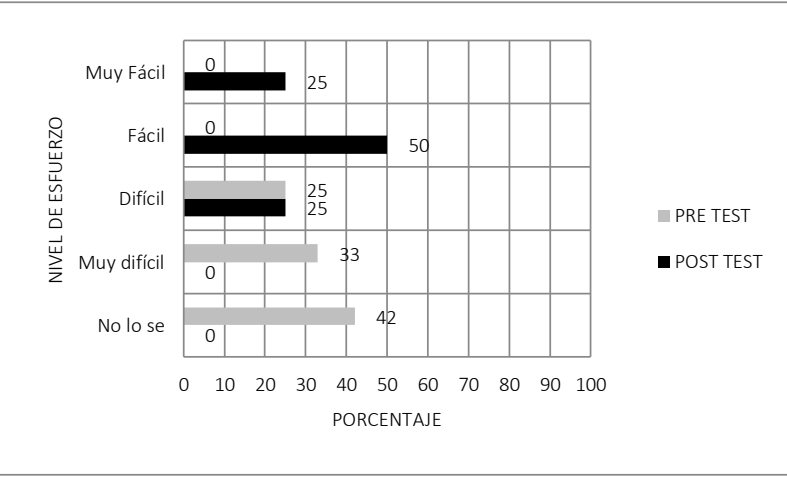

Figura 7.Empaquetar el OA.

Las figuras 6 y 7 muestran claramente que los docentes inicialmente o no conocían o no opinaban que pudieran realizar tareas ligadas al ensamblar o empaquetar un OA. Y aunque después del curso sigue habiendo algunos que consideran estas actividades difíciles, las respuestas en el pretest muestran un cambio en sus opiniones debido al entrenamiento proporcionado en el curso. 


\subsection{Preguntas abiertas al inicio y al final del curso, agregadas al cuestionario}

Las preguntas abiertas sirvieron como complemento al cuestionario con el objetivo de confirmar los hallazgos. A continuación, las respuestas de los participantes y el tipo de fase en el diseño de un OA a la que aluden.

Tabla I. Preguntas abiertas hechas al inicio y al final del curso. Elaboración propia.

\begin{tabular}{|c|c|c|c|c|}
\hline \multirow{3}{*}{$\begin{array}{l}\text { 岁 } \\
\text { 岁 }\end{array}$} & \multicolumn{2}{|c|}{ PREGUNTA ABIERTA INICIO } & \multicolumn{2}{|c|}{ PREGUNTA ABIERTA FINAL } \\
\hline & \multicolumn{2}{|c|}{$\begin{array}{l}\text { De las siguientes actividades escriba por qué algunas le } \\
\text { resultan tareas fáciles y otras difíciles al diseñar un RED: } \\
\text { - Definir el objetivo del recurso y estudiante al que va dirigido. } \\
\text { - Definir estructura del recurso. } \\
\text { - Crear los textos, gráficos, audios del recurso. } \\
\text { - Hacer funcionar el recurso. }\end{array}$} & \multicolumn{2}{|c|}{$\begin{array}{l}\text { ¿Cuáles fases en el diseño de su OA le } \\
\text { resultaron más fáciles y cuáles más difíciles? }\end{array}$} \\
\hline & FÁCIL & DIFÍCIL & FÁCIL & DIFÍCIL \\
\hline 1 & \multicolumn{2}{|c|}{ SR (SIN RESPUESTA) } & $\begin{array}{l}\text { "la evaluación..." } \\
\text { [Evaluación] }\end{array}$ & $\begin{array}{l}\text { "Difícil y enriquecedora, } \\
\text { la implementación." } \\
\text { [Implementación] }\end{array}$ \\
\hline 2 & $\begin{array}{l}\text { "definir el objetivo del curso, a } \\
\text { quién está dirigido, hacer los } \\
\text { textos y gráficos." } \\
\text { [Análisis, } \\
\text { Diseño Multimedial] }\end{array}$ & $\begin{array}{l}\text { "hacer funcionar el recurso } \\
\text { y hacer los audios, porque } \\
\text { tienen que ver con mi } \\
\text { habilidad para acceder a } \\
\text { plataformas o conocer } \\
\text { aplicaciones que me } \\
\text { permitan aterrizar lo que } \\
\text { tengo en mente." } \\
\text { [Diseño Multimedial } \\
\text { Implementación] }\end{array}$ & $\begin{array}{l}\text { "Fáciles: } \\
\text { determinar los } \\
\text { objetivos, } \\
\text { contenidos y } \\
\text { jerarquías." } \\
\text { [Análisis, } \\
\text { Diseño } \\
\text { Instruccional] }\end{array}$ & $\begin{array}{l}\text { "Difícil: diseñar } \\
\text { actividades y } \\
\text { evaluaciones útiles } \\
\text { acordes a los estilos de } \\
\text { aprendizaje y } \\
\text { compatibles con la } \\
\text { plataforma, migrar a lo } \\
\text { virtual los materiales y } \\
\text { diseñar de forma } \\
\text { uniforme los mismos." } \\
\text { [Diseño Instruccional, } \\
\text { Diseño Multimedial, } \\
\text { Implementación] }\end{array}$ \\
\hline 3 & \multicolumn{2}{|c|}{ SR } & $\begin{array}{l}\text { "Fácil, la } \\
\text { planeación..." } \\
\text { [Análisis, } \\
\text { Diseño } \\
\text { Instruccional] }\end{array}$ & $\begin{array}{l}\text { "...difícil: la creación." } \\
\text { [Diseño instruccional } \\
\text { Implementación] }\end{array}$ \\
\hline 4 & SR & $\begin{array}{l}\text { "Mi dificultad estriba en el } \\
\text { poco conocimiento que } \\
\text { tengo de los recursos y me } \\
\text { resulta fácil el diseño una } \\
\text { vez conociendo las } \\
\text { características, cualidades, } \\
\text { etc. del recurso. Es decir, } \\
\text { los aspectos técnicos de los } \\
\text { recursos digitales se me } \\
\text { dificultan porque los } \\
\text { desconozco." }\end{array}$ & $\begin{array}{l}\text { "Se me facilitó el } \\
\text { área } \\
\text { instruccional,.." } \\
\text { [Análisis, } \\
\text { Diseño } \\
\text { Instruccional] }\end{array}$ & $\begin{array}{l}\text { “...se me complicó la } \\
\text { tecnológica." } \\
\text { [Diseño Multimedial } \\
\text { Implementación] }\end{array}$ \\
\hline
\end{tabular}




\begin{tabular}{|c|c|c|c|c|}
\hline & & [Implementación] & & \\
\hline 5 & $\begin{array}{l}\text { "fáciles porque interactúo } \\
\text { frecuentemente con } \\
\text { aplicaciones tecnológicas." } \\
\text { [Todas las fases fáciles] }\end{array}$ & SR & $\begin{array}{l}\text { "Fácil - definición } \\
\text { del OA..." } \\
\text { [Análisis, } \\
\text { Diseño] }\end{array}$ & $\begin{array}{l}\text { "...díficil - la } \\
\text { implementación." } \\
\text { [Implementación] }\end{array}$ \\
\hline 6 & $\begin{array}{l}\text { "Definir objetivo del recurso y } \\
\text { estudiante al que va dirigido: } \\
\text { fácil, me enfoco en las } \\
\text { necesidades del programa. } \\
\text { Definir estructura: fácil: } \\
\text { responde a las necesidades de } \\
\text { programa. Crear textos: fácil, se } \\
\text { crean en colectivo.Crear } \\
\text { gráficos: fácil, porque uso pocos } \\
\text { datos. Crear audios del recurso: } \\
\text { fácil, si se da el caso, son } \\
\text { recursos de la red. Hacer } \\
\text { funcionar el recurso: fácil si hay } \\
\text { conectividad, difícil si no la hay." } \\
\text { [Todas las fases fáciles] }\end{array}$ & SR & $\begin{array}{l}\text { "Definir el } \\
\text { objetivo, } \\
\text { seleccionar los } \\
\text { contenidos y } \\
\text { elegir el estilo de } \\
\text { aprendizaje fue } \\
\text { fácil." } \\
\text { [Análisis, } \\
\text { Diseño } \\
\text { Instruccional] }\end{array}$ & $\begin{array}{l}\text { "Descargar el } \\
\text { Exelearning y } \\
\text { familiarizarme con el } \\
\text { programa fue difícil." } \\
\text { [Implementación] }\end{array}$ \\
\hline 7 & $S R$ & $\begin{array}{l}\text { "difícil crear gráficos y } \\
\text { audios ya que no conozco } \\
\text { muchas herramientas para } \\
\text { ello." } \\
\text { [Diseño Multimedial, } \\
\text { Implementación] }\end{array}$ & $\begin{array}{l}\text { "no hubo un tema } \\
\text { difícil, ya que } \\
\text { contábamos con } \\
\text { todo el material } \\
\text { suficiente para } \\
\text { hacerlo." } \\
\text { [Todas las } \\
\text { opciones fáciles] }\end{array}$ & SR \\
\hline 8 & $\begin{array}{l}\text { "Definir el objetivo y estudiante } \\
\text { al que va dirigido creo que será } \\
\text { fácil dado que estoy } \\
\text { familiarizada (aunque no de } \\
\text { manera digital)." } \\
\text { [Análisis] }\end{array}$ & $\begin{array}{l}\text { "Crear gráficos, audios del } \\
\text { recurso tal vez sea el reto } \\
\text { porque no soy experta en } \\
\text { el uso de herramientas } \\
\text { técnológicas." } \\
\text { [Diseño Multimedial, } \\
\text { Implementación] }\end{array}$ & $S R$ & $\begin{array}{l}\text { "...el diseño multimedia } \\
\text { fue difícil para mi, faltó } \\
\text { tiempo para explorar el } \\
\text { programa Exelerning y } \\
\text { sacar más provecho de } \\
\text { las herramientas, no } \\
\text { pude explorar todas." } \\
\text { [Diseño Multimedial, } \\
\text { Implementación] }\end{array}$ \\
\hline 9 & $\begin{array}{l}\text { "Crear los textos, gráficos, } \\
\text { audios del recurso: se me facilita } \\
\text { ya que tuve un curso en la } \\
\text { maestría para crear este tipo de } \\
\text { recursos. } \\
\text { Hacer funcionar el recurso: se } \\
\text { me facilita ya que conozco la } \\
\text { plataforma y los medios que } \\
\text { puedo usar." } \\
\text { [Diseño Multimedial, } \\
\text { Implementación] }\end{array}$ & $\begin{array}{l}\text { "Definir el objetivo del } \\
\text { recurso y estudiante al que } \\
\text { va dirigido: se me complica } \\
\text { ya que a veces no logro } \\
\text { separar estos objetivos. } \\
\text { Definir estructura del } \\
\text { recurso: se me complica ya } \\
\text { que en ocasiones saturo } \\
\text { con demasiadas actividades } \\
\text { o documentos la } \\
\text { plataforma." } \\
\text { [Diseño Instruccional] }\end{array}$ & SR & $\begin{array}{l}\text { "No fueron difíciles, sin } \\
\text { embargo, destacaría que } \\
\text { la última fase, de } \\
\text { implementación, se me } \\
\text { complicó debido al nivel } \\
\text { de detalle y de cuidado } \\
\text { que debía tener." } \\
\text { [Implementación] }\end{array}$ \\
\hline 10 & $\begin{array}{l}\text { "Definir el objetivo del recurso y } \\
\text { estudiante al que va dirigido / } \\
\text { definir estructura del recurso: } \\
\text { han resultado ser fáciles debido } \\
\text { a que no han presentado } \\
\text { complicación alguna en } \\
\text { transmitir a los estudiantes }\end{array}$ & $x^{2}$ & $\begin{array}{l}\text { "Considero que } \\
\text { todas las fases } \\
\text { fueron sencillas ya } \\
\text { que estuvieron } \\
\text { bien explicadas." } \\
\text { [Todas fases } \\
\text { fáciles] }\end{array}$ & SR \\
\hline
\end{tabular}




\begin{tabular}{|c|c|c|c|c|}
\hline & $\begin{array}{l}\text { aquello que se busca, se espera } \\
\text { de tales recursos." }\end{array}$ & & & \\
\hline 11 & SR & $\begin{array}{l}\text { "...y es difícil crear gráficos } \\
\text { porque hay que dedicarle } \\
\text { tiempo." } \\
\text { [Diseño Multimedial] }\end{array}$ & $\begin{array}{l}\text { "Fácil el de } \\
\text { Análisis." } \\
\text { [Análisis] }\end{array}$ & $\begin{array}{l}\text { "Difícil el Módulo de } \\
\text { Implementación." } \\
\text { [Implementación] }\end{array}$ \\
\hline 12 & $\begin{array}{l}\text { "Me parece fácil definir el } \\
\text { objetivo, estudiante al que va } \\
\text { dirigido y estructura del recurso } \\
\text { porque al conocer el contenido } \\
\text { y objetivo de la materia se } \\
\text { establecen sin problemas." } \\
\text { [Análisis, } \\
\text { Diseño Instruccional] }\end{array}$ & $\begin{array}{l}\text { "Me parece difícil crear } \\
\text { audios, gráficos o hacer } \\
\text { funcionar el recurso por mi } \\
\text { poco acercamiento a la } \\
\text { tecnología o plataformas } \\
\text { especializadas para ello." } \\
\text { [Diseño Multimedial, } \\
\text { Implementación] }\end{array}$ & $S R$ & $S R$ \\
\hline
\end{tabular}

La tabla I permite apreciar que en el caso de la pregunta inicial 5 de los participantes no dieron respuesta a lo que consideraban fácil y los 5 que lo hicieron señalaron la definición de un objetivo incluido en las tareas de Análisis de la fase 1 en la elaboración del OA. En el caso de lo difícil en números semejantes los docentes señalaron o el diseño multimedial o la implementación, mostrando así su desconocimiento de la mayoría de las tareas incluidas en la metodología que sería utilizada en el diseño del OA y sus dificultades en aspectos técnicos de los recursos digitales. Para el caso de la pregunta final los profesores que respondieron acerca de lo que se les hacia fácil todos mencionaron que la fase de análisis, el diseño instruccional y/o todas las fases, mostrando que el curso les había ayudado y reafirmando los datos obtenidos en la encuesta de opinión arriba presentada. Finalmente es de señalar que 9 de los 12 docentes consideraron como difícil aún después del curso la implementación del OA, nuevamente indicando lo complejo de los aspectos tecnológicos en sus diseños de OA. Los argumentos esgrimidos confirman lo observado en los resultados de las opiniones iniciales y finales de los participantes presentadas en las preguntas cerradas del cuestionario.

\section{CONCLUSIONES}

- La experiencia de acompañamiento y codiseño de OA con los docentes de la muestra que esta investigación presenta, funcionó para identificar cuáles son las áreas en las que el docente requiere de más herramientas, apoyo o capacitación y cumplió con el objetivo planteado, de ser una estrategia de análisis de metodologías específicas en nuestro caso DICREVOA 2.0 y aplicaciones de acompañamiento y codiseño y una situación que propiciara el aprendizaje de competencias y actualización de los docentes en recursos didácticos y en particular digitales.

- Los datos obtenidos permiten apreciar lo incipiente de las capacidades para diseñar un OA en docentes de la muestra seleccionada y en la elaboración de recursos educativos y la posibilidad de mejora de las mismas mediante un curso de acompañamiento y codiseño en la elaboración de un OA. Igualmente queda evidenciada la necesidad de mayor preparación y acompañamiento a los docentes en el manejo de competencias ligadas al uso pedagógico y tecnológico requerido por la educación actual y futura, como ya ha sido señalado con anterioridad (Mendieta-Baltodano, Vázquez-Cano y Cobos-Sanchiz, 2017). 
Cabe señalar que dado el tamaño de la muestra los resultados obtenidos no permiten realizar generalizaciones y requieren de futuros estudios al respecto.

- Los aspectos tecnológicos implicados en la elaboración de un OA y por consiguiente quizá de cualquier (RED) de este tipo, inicialmente le son totalmente desconocidos a los participantes. Cabe enfatizar que el curso tuvo una duración de solo 25 horas y que la información y la ejecución de las tareas no daban mucho tiempo al docente para completarlas holgadamente, sino le exigían que trabajara eficientemente en este periodo. Creemos que los datos iniciales al compararlos con los posteriores al curso, pudiesen haber sido diferentes y mejores con un curso de más larga duración, que hubiera permitido mayor práctica. Sin embargo, estas son las condiciones reales que tienen los docentes universitarios para capacitarse y mejorar su práctica.

- En general los resultados apuntan a la necesidad de realizar mayor investigación sobre estas temáticas para generar propuestas y soluciones en la educación superior. Para finalizar, la contingencia actual del COVID 19 y su impacto global, está haciendo que, para poder transmitir conocimiento en las instituciones educativas, los docentes se tengan que enfrentar a los retos del uso de la TIC de manera acelerada y obligatoria. Un estudio como el aquí descrito, en fechas posteriores, seguramente mostrará cambios, que las exigencias de la vida real les han planteado a los docentes para seguir haciendo su labor profesional, incrementado sus competencias y modificado sus actitudes.

\section{REFERENCIAS}

Barba, M., De la Calle, L., Calderón, E. y Ramos, Y. (2019). Capacitación en el diseño de objetos de aprendizaje para la actualización en recursos didácticos con sustento en la Tic. Pro Sciences: Revista De Producción, Ciencias e Investigación, 3(29), 9-19. https://doi.org/10.29018/issn.2588-1000vol3iss29.2019pp9-19

Cabero, J. (2014). La formación del profesorado en TIC: modelo TPACK. España: Secretariado de recursos Audiovisuales y Nuevas Tecnologías de la Universidad de Sevilla.

Camargo-Escobar, I., y Pardo-Adames, C. (2008). Competencias docentes de profesores de pregrado: diseño y validación de un instrumento de evaluación. Universitas Psychologica, 7(2), 441-457.

Casadei, L., Barrios, I. (2016). Determinación de las competencias docentes para el desarrollo de recursos educativos digitales. Eduweb, Revista de Tecnología de Información y $\begin{array}{llll}\text { Comunicación en 25-40. } & \text { 10(1), Educación, }\end{array}$ https://dialnet.unirioja.es/servlet/articulo?codigo=5905341

CODAES (2015). Guía OA-CODAES. CODAES. http://www.codaes.mx/content/micrositios/2/file/GuiaOA-CODAES.pdf

Gordillo, A., López-Pernas, S., y Barra, E. (2019). Efectividad de los MOOC para docentes en el uso seguro de las TIC. Comunicar, 61, 103-112. https://doi.org/10.3916/C61-2019-09 
Maldonado, J., Sanz, C., Fernández-Pampillón y Astudillo, A. (2015). Desarrollo de un Marco de Análisis para la Selección de Metodologías de Diseño de Objetos de Aprendizaje (OA) basado en criterios de calidad para contextos educativos específicos (Tesis Doctoral). Universidad Nacional de la Plata, Facultad de Informática. La Plata, Buenos Aires, Argentina.

Maldonado, J., Bermeo, J. y Vélez, F. (2017). Diseño, Creación y Evaluación de Objetos de Aprendizaje. Metodología DICREVOA 2.0. CEDIA.

Martínez-Garcés, J. y Garcés-Fuenmayor, J. (2020). Competencias digitales docentes y el reto de la educación virtual derivado de la covid19. Educación y Humanismo, 22(39), 1-16. https://doi.org/10.17081/eduhum.22.39.4114

Mendieta-Baltodano, C. de M., Vázquez-Cano, E., y Cobos-Sanchiz, D. (2017). Valoración de las competencias tecnológicas del profesorado universitario. Edutec. Revista $\begin{array}{llll}\text { Electrónica De Tecnología } & \text { (60). }\end{array}$ https://doi.org/10.21556/edutec.2017.60.843

Menéndez, V., Prieto, M. y Zapata, A. (2010). Sistemas de Gestión Integral de Objetos de Aprendizaje. IEEE-RITA, 5(2). http://rita.det.uvigo.es/201005/uploads/IEEERITA.2010.V5.N2.A3.pdf

Morales, E., García, F., Moreira, T., Rego, H. y Berlanga, A. (2005). Valoración de la calidad de unidades de aprendizaje. Revista De Educación a Distancia (RED), Monográfico III. 1-13 https://revistas.um.es/red/article/view/24571

Morales Velasco, R. A., y Diez-Martinez Day, E. (2020). Revisión de metodologías para diseñar Objetos de Aprendizaje OA: un apoyo para docentes. Revista Iberoamericana de Tecnología En Educación y Educación En Tecnología, (26 SE-), e4. https://doi.org/10.24215/18509959.26.e4

Ogalde, I. y González, M. (2008). Nuevas Tecnologías y Educación. Diseño, desarrollo, uso y evaluación de materiales educativos. Trillas.

Trujillo, L. (2013). La construcción de materiales educativos, una competencia para el docente del siglo XXI. Ponencia en: XIV Encuentro Internacional Virtual Educa. Foro multilateral, congreso, exposición educación, innovación y TIC. Medellín. https://reposital.cuaed.unam.mx:8443/xmlui/handle/123456789/3741

\section{Para citar este artículo:}

Diez-Martinez, E. y Morales-Velasco, R. A. (2020). Codiseño de Objetos de Aprendizaje OA como estrategia de capacitación a docentes de Educación Superior . Edutec. Revista Electrónica De Tecnología Educativa, (74), 114-126. https://doi.org/10.21556/edutec.2020.74.1765 